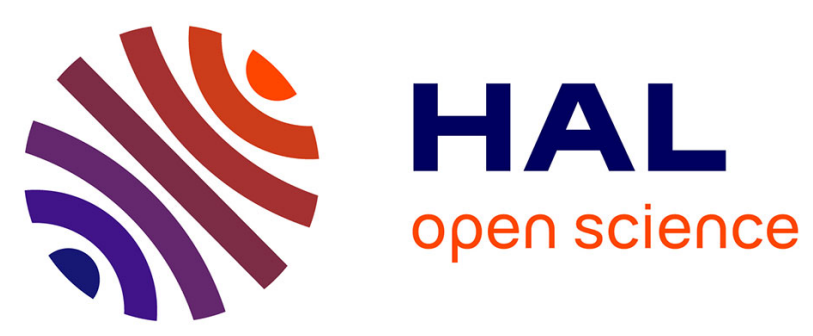

\title{
La production d'ignifracts par l'incendie du 28 août 1989 sur les parois de la montagne Sainte-Victoire
}

\author{
Jean-Louis Ballais, Marie-Christine Bosc
}

\section{To cite this version:}

Jean-Louis Ballais, Marie-Christine Bosc. La production d'ignifracts par l'incendie du 28 août 1989 sur les parois de la montagne Sainte-Victoire. Méditerranée: revue géographique des pays méditerranéens, 1992, Sainte-Victoire, hommes et paysages, 75 (1.2), pp.53-58. 10.3406/medit.1992.2753 . hal-01562637

\section{HAL Id: hal-01562637 https://hal-amu.archives-ouvertes.fr/hal-01562637}

Submitted on 20 Jul 2017

HAL is a multi-disciplinary open access archive for the deposit and dissemination of scientific research documents, whether they are published or not. The documents may come from teaching and research institutions in France or abroad, or from public or private research centers.
L'archive ouverte pluridisciplinaire HAL, est destinée au dépôt et à la diffusion de documents scientifiques de niveau recherche, publiés ou non, émanant des établissements d'enseignement et de recherche français ou étrangers, des laboratoires publics ou privés. 


\section{La production d'ignifracts par l'incendie du 28 Août 1989 sur les} parois de la montagne Sainte-Victoire

In: Méditerranée, Tome 75, 1-2-1992. Sainte-Victoire, hommes et paysages. pp. 53-58.

Citer ce document / Cite this document :

Ballais Jean-Louis, Bosc Marie-Chritine. La production d'ignifracts par l'incendie du 28 Août 1989 sur les parois de la montagne Sainte-Victoire. In: Méditerranée, Tome 75, 1-2-1992. Sainte-Victoire, hommes et paysages. pp. 53-58.

doi : 10.3406/medit.1992.2753

http://www.persee.fr/web/revues/home/prescript/article/medit_0025-8296_1992_num_75_1_2753 


\section{La production d'ignifracts par l'incendie du 28 août 1989 sur les parois de la montagne Sainte-Victoire}

\section{Jean-Louis BALLAIS*, Marie-Christine BOSC**}

Le 28 août 1989, à 11 h 43 mn, le feu se déclare à l'extrémité occidentale de la montagne SainteVictoire, à l'est d'Aix-en-Provence (fig. 1). Le mistral soufflait à $70-80 \mathrm{~km} / \mathrm{h}$ avec des rafales atteignant $100 \mathrm{~km} / \mathrm{h}$ et la réserve d'eau du sol était tombée en-dessous de $10 \mathrm{~mm}$ depuis plusieurs jours déjà. Malgré l'intervention rapide des pompiers, en faction à seulement $2 \mathrm{~km}$, les conditions étaient tellement favorables qu'il a été impossible d'empêcher la propagation du feu. En un peu plus de $3 \mathrm{~h}$, tout le versant méridional de la montagne était dévasté par l'incendie. En tout, c'est environ 5000 ha de garrigues et de forêts claires de Pinus halepensis qui ont brûlé (BACCOUCHE, 1990).

\section{1 - LES IGNIFRACTS}

Dès les premiers jours de septembre, nous avons pu observer que les parois et les bancs rocheux (calcaires jurassiques, brèches et conglomérats du Crétacé supérieur, de l'Eocène et du Quaternaire) commençaient à s'écailler. A leur pied s'accumulaient des éclats très minces, fragiles, parfois déjà fragmentés, noircis par le feu sur la face externe et laissant apparaître la structure de la roche sur la face interne. Générés par le passage du feu, nous proposons de les nommer ignifracts (du latin ignis : feu) et ignifraction le processus qui les a engendrés (photo 1).

\section{1 - Caractérisation statistique}

Pour caractériser ces objets et pouvoir les comparer avec d'autres éclats (gélifracts ou thermoclasts), nous en avons fait une étude statistique préliminaire, en particulier celle de leur indice d'aplatissement [Ia $=L+l / 2 e$, où $L$ est la longueur, $l$ la largeur et $e$ l'épaisseur (TRICART, CAILLEUX, 1955)]. 162 ignifracts ont été prélevés sur une faible superficie, dans le petit bassin-versant $\left(3,1 \mathrm{~km}^{2}\right)$ du ruisseau des Deux-Aiguilles, juste à l'est de l'oppidum de Saint-Antonin (fig. 1). L'ignifract moyen prélevé mesure $109,2 \mathrm{~mm} \times 77,9 \mathrm{~mm} \times 18,1 \mathrm{~mm}$ pour un poids d'environ $385 \mathrm{~g}$.

En fait, les longueurs varient entre $188,5 \mathrm{~mm}$ et $41 \mathrm{~mm}$, les largeurs entre $154 \mathrm{~mm}$ et $27 \mathrm{~mm}$ et les épaisseurs entre $44 \mathrm{~mm}$ et $5 \mathrm{~mm}$. Surtout, l'échantillonnage ne rend pas exactement compte de l'hétérométrie des ignifracts car ni les plus petits, trop fragiles pour être déplacés, ni les plus grands, trop lourds pour être transportés, ont été décomptés.

L'indice moyen d'aplatissement est de 5,6. C'est un chiffre beaucoup plus élevé que celui des gélifracts (2,5 selon TRICART, CAILLEUX, 1955). Mieux, l'indice minimum observé sur notre échantillonnage, c'est-à-dire 2,88, est déjà supérieur à l'aplatissement moyen dû à la gélifraction.

* UFR des Sciences Géographiques et de l'Aménagement, Université d'Aix-Marseille II et URA 903 CNRS.

** UFR des Sciences Géographiques et de l'Aménagement, Université d'Aix-Marseille II. 


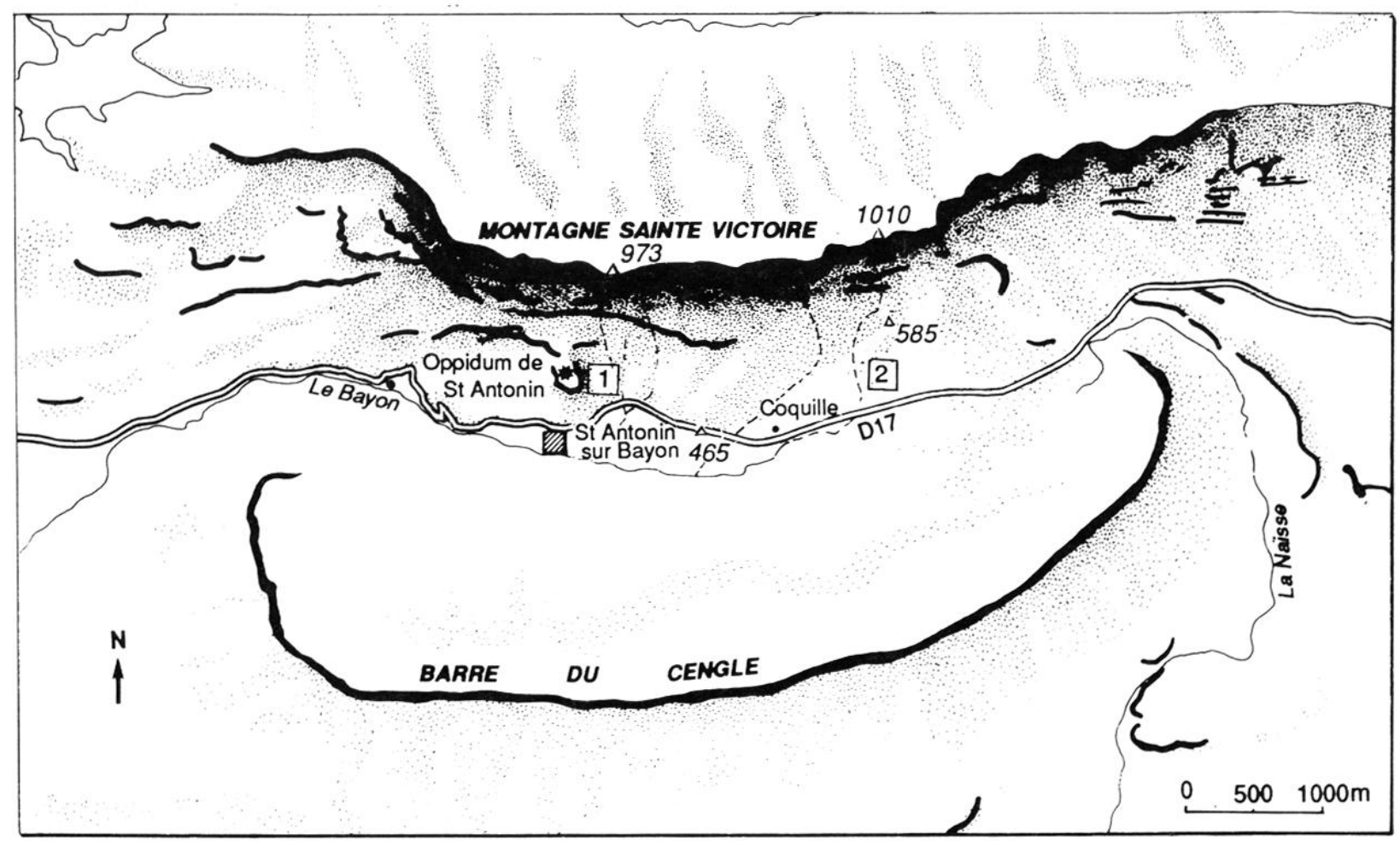

Fig. 1 - CROQUIS DE LOCALISATION

1: Ruisseau des Deux-Aiguilles ; 2 : Ruisseau de Roque-Vaoutade

Statistiquement, la confusion entre ignifracts et gélifracts n'est pas possible : l'ignifraction fournit un matériel original et qui peut être identifié facilement par son indice d'aplatissement.

L'analyse factorielle montre que la longueur, la largeur et l'épaisseur se corrèlent bien : coefficient de 0,842 entre la longueur et la largeur, 0,716 entre la longueur et l'épaisseur, 0,661 entre la largeur et l'épaisseur : plus un ignifract est long, plus il est large et épais. Il n'est donc pas surprenant que l'indice d'aplatissement ne se corrèle ni avec la longueur (coefficient de - 0,017), ni avec la largeur (coefficient de 0,020). La meilleure corrélation se fait avec l'épaisseur (coefficient de 0,596) qui a la corrélation la moins forte avec la longueur.

La matrice factorielle confirme bien ces résultats. On y remarque que le premier facteur extrait regroupe les trois mesures de dimension $(0,90239$ pour la longueur, 0,87599 pour la largeur et 0,92228 pour l'épaisseur), mettant à part l'indice d'aplatissement (- 0,35210). Alors que pour le second facteur, c'est le contraire : il est fortement lié à l'indice d'aplatissement $(0,92561)$ et très peu à la longueur $(0,32721)$, à la largeur $(0,37646)$ et à l'épaisseur $(-0,32424)$.

\section{2 - L'Influence de la lithologie}

En l'absence de tout lien entre les dimensions et l'aplatissement des ignifracts, il fallait rechercher le rôle éventuel de la nature lithologique des échantillons. Afin de pouvoir introduire numériquement ce nouveau paramètre, nous avons établi un second fichier, à matrice booléenne, sur lequel nous avons effectué les mêmes opérations que sur le premier fichier. L'extraction de deux facteurs seulement, dont le premier regroupait les trois mesures de dimension, nous a amenés à ne conserver que l'une des trois dans le nouveau fichier : c'est la longueur que nous avons retenue. En outre, la délimitation des trois catégories de longueur (court, moyen, long) et des trois catégories d'aplatissement (peu aplati, moyennement aplati, très aplati) s'est basée sur les premiers résultats. Dans les deux cas, la première limite a été fixée à $x-1 / 2 \sigma x$ et la deuxième à $x+1 / 2 \sigma x$, où $x$ est la moyenne et $\sigma$ l'écart-type. Nous pouvons ainsi répartir les objets en ignifracts courts $(28,4 \%)$, moyens $(44,4 \%)$ et longs $(27,2 \%)$. La catégorie la plus nombreuse est donc la moyenne, tandis que les deux autres se répartissent presqu'également le reste des objets. La répartition est donc symétrique par rapport à la catégorie moyenne.

Ce n'est plus le cas lorsqu'on étudie l'aplatissement, puisqu'il y a $37,7 \%$ de peu aplatis, $35,2 \%$ de moyennement aplatis et $27,2 \%$ de très aplatis. On observe là que les deux premières catégories comptent 
chacune un peu plus du tiers des objets, alors que la troisième est moins abondante. En outre, la catégorie des peu aplatis excède légèrement celle des moyennement aplatis.

Ces résultats indiquent que si la moyenne des longueurs, calculée sur le premier fichier, offre un bon résumé de cette mesure, ce n'est pas le cas de la moyenne des indices d'aplatissement. Celle-ci est décentrée vers le bas, dans l'intervalle des indices calculés $(2,88 ; 10,33)$.

Mais sur ce deuxième fichier, l'analyse factorielle donne des résultats bien plus décisifs. Le tableau de corrélation montre qu'il n'y a aucun lien entre la lithologie et l'aplatissement. En effet, les coefficients de corrélation entre les deux variables lithologiques (calcaire et brèches à ciment calcaire) et les trois de l'aplatissement oscillent entre $-7,5 \cdot 10^{3}$ et $7,5.10^{3}$. C'est dire qu'ils sont dérisoires et qu'il n'y a aucune relation entre la lithologie et l'aplatissement. C'est un résultat très inattendu, contraire à ce qui est admis pour les gélifracts.

Outre cette conclusion fondamentale, la lecture du tableau de corrélation fait aussi ressortir un lien entre la lithologie et les ignifracts courts, lien confirmé par les tableaux Factor Matrix et Eigenvectors. Ils font ressortir une corrélation entre les variables «long» et «très aplati», d'une part, et entre les variables «moyen» et «peu aplati» d'autre part, liens qui n'apparaissaient que faiblement dans le tableau de corrélation. De fait, le premier facteur, qui représente près du tiers de la variance totale, accentue la corrélation entre le matériel court $(\mathfrak{f} 2)$ et les brèches $(\mathfrak{f} 9)$ et la corrélation inverse entre le matériel court et le calcaire ( $£$ 8). On peut donc définir une catégorie d'ignifracts courts et bréchiques.

Le second facteur, qui ajouté au premier représente $55,09 \%$ de la variance totale, fait se corréler les catégories moyennement aplatis $(-0,77167)$ et peu aplatis $(-0,72211)$. On peut donc définir une deuxième catégorie d'ignifracts de taille moyenne et peu aplatis.

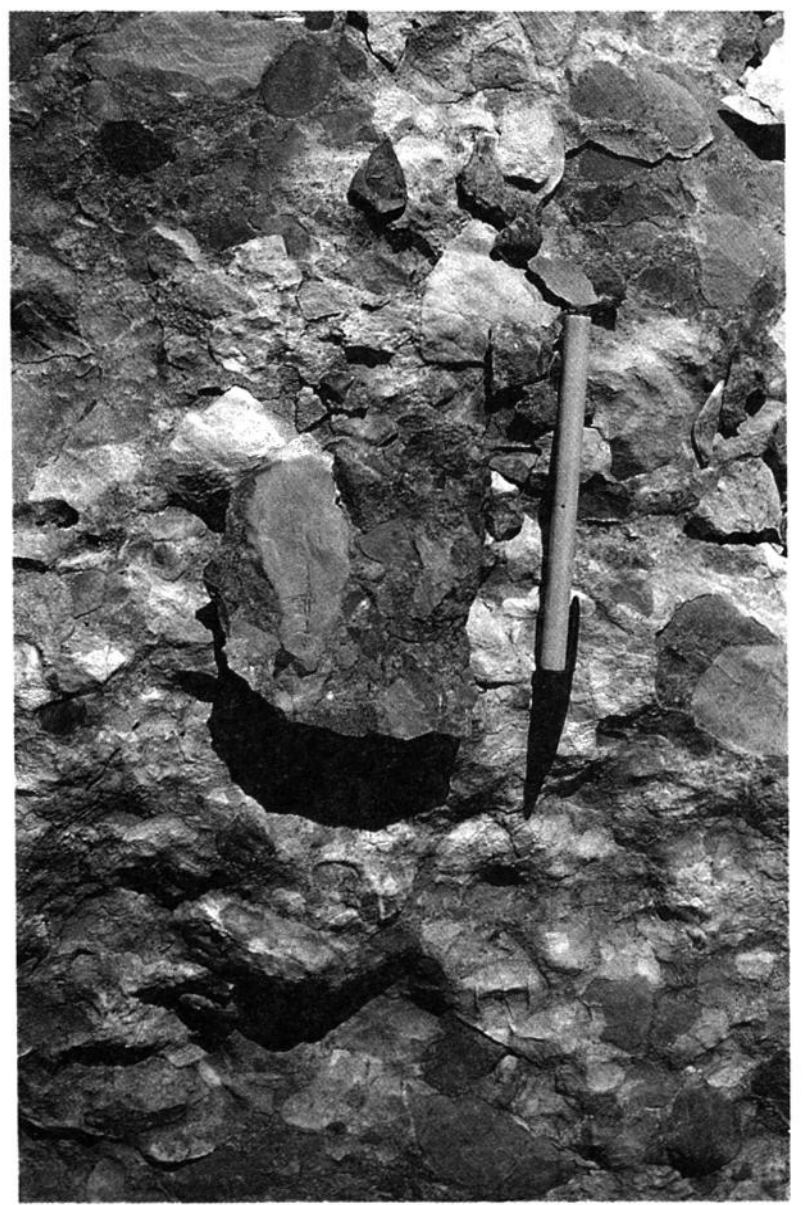

Photo 1 - IGNIFRACT DÉTACHÉ D'UN BANC DE BRĖCHES. (Cliché : J.L. BALLAIS)

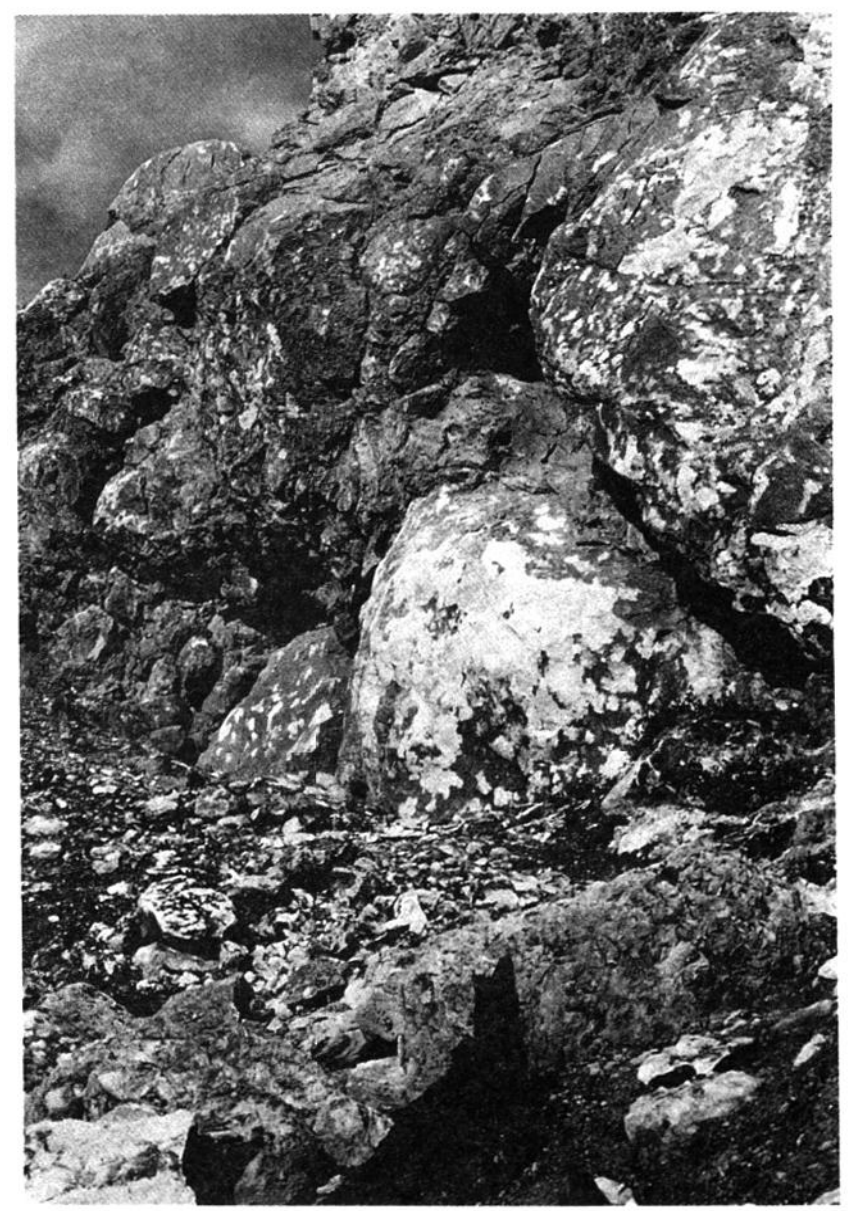

Photo 2 - PAROI IGNIFRACTEE. LA SURFACE IGNIFRACTÉE SE DETTACHE EN CLAIR SUR LA PAROI NOIRCIE PAR LE FEU. (Cliché : J.L. BALLAIS) 


\section{3 - Conclusions}

Ce traitement statistique fournit donc une connaissance substantielle de la structure de l'échantillonnage. Il permet d'isoler deux catégories bien délimitées : celle des petits ignifracts sur brèches et celle des ignifracts moyens peu aplatis. Néanmoins, tous les liens entre les variables du second fichier restent médiocres. L'extraction de cinq facteurs, dont aucun ne représente une grande part de la variance totale, alors que le facteur 1 de la première analyse factorielle faisait quelques $63 \%$ de la variance totale et que les deux facteurs réunis en faisaient un peu plus de $94 \%$, confirme bien que le deuxième fichier ne présente pas une structure très cohérente. Les deux catégories, petits ignifracts sur brèches et ignifracts moyens peu aplatis, sont loin de se répartir l'ensemble des 162 échantillons. Ils constituent les deux grands types d'objets statistiquement identifiables, mais le reste de l'échantillonnage demeure mal structuré, difficile à appréhender et à classer.

Surtout, le traitement statistique permet de rejeter l'hypothèse de l'influence des dimensions des ignifracts sur leur indice d'aplatissement ainsi que celle de l'influence de la lithologie sur ce même indice, contribuant ainsi à une caractérisation accrue de ces objets.

\section{2 - L'ABLATION PAR IGNIFRACTION}

\section{1 - L'évolution temporelle après l'incendie}

Dès les premiers jours après l'incendie, des chutes d'ignifracts ont été observées. Comme pour les autres processus de fragmentation mécanique, 1 'intervale de temps entre l'individualisation des ignifracts et leur détachement de la paroi suivi de leur chute a été très variable.

Au cours des premières semaines de l'automne 1989, les pluies, quoique peu intenses (BOSC, 1990), ont favorisé les chutes. Le vent, le mistral en particulier, a très probablement joué un rôle qu'il n'est pas possible de préciser plus.

Au cours de l'hiver, deux nouveaux processus sont venus s'ajouter aux précédents. Tout d'abord, la gélifraction. Très modérée en raison du petit nombre de cycles gel-dégel d'un hiver particulièrement doux, elle a cependant favorisé efficacement le détachement des ignifracts car l'eau pouvait facilement s'infiltrer entre eux et la paroi rocheuse. L'autre processus est typiquement anthropique, combinaison de «purge» volontaire et de détachement involontaire sous les pieds des escaladeurs, dès avant que les autorités préfectorales eurent donné l'autorisation de réutiliser les voies d'escalades habituelles très fréquentées dans le bassin-versant du ruisseau des Deux-Aiguilles (photo 2).

Il semble bien que la plus grande partie des ignifracts ait été mobilisée au cours de cet hiver 1989-1990. Depuis, les détachements paraissent très localisés, mais les zones de départ continuent à rester très visibles car la patine ne s'est pas encore reformée sur les affleurements rocheux (photo 2).

\section{2 - Répartition, cause et importance de l'Ignifraction}

Au premier abord, la répartition des ignifracts paraît très irrégulière. Cependant, leur densité est maximum sur les parois subverticales et dans les premiers mètres au-dessus du sol. A une échelle plus fine, c'est à nouveau la variabilité qui domine : une paroi a pu être affectée dans une proportion de 50\% en un point, et dans une proportion de quelques \% à quelques mètres de là, voire ne pas avoir été affectée du tout.

L'hypothèse la plus vraisemblable pour expliquer ces variations est évidemment les caractéristiques du feu. Rappelons en effet que, à échelle macroscopique, ni la localisation des ignifracts en surface, ni leur surface de détachement, rugueuse, avec la roche en place, ne semblent influencées par les caractéristiques de la patine ou par une éventuelle altération superficielle. L'ignifraction doit donc être considérée comme une forme particulière de thermoclastie. 
D'après les témoignages visuels des pompiers, l'incendie ne présentait pas un front uniforme et la température à laquelle il a porté superficiellement les parois a dû varier considérablement d'un point à un autre, en fonction de la densité du boisement notamment. De même, le temps pendant lequel les températures sont restées élevées a varié considérablement. Il arrivait même qu'un petit périmètre soit épargné à la faveur des bourrasques du mistral. Cependant, des observations supplémentaires, certes difficiles, seront nécessaires car l'observation des parois, quelques semaines après l'incendie n'a pas montré de corrélation claire entre, par exemple, les bouquets de Pinus halepensis et les densités d'ignifracts.

Il ne faut pas négliger non plus, en effet, le rôle de la structure des roches cohérentes : changements latéraux de faciès, surtout dans les brèches, mais aussi fragmentation due à la tectonique, particulièrement vigoureuse sur ce front de chevauchement.

Faute d'une étude détaillée, il n'est pas possible de chiffrer avec précision le tonnage représenté par les ignifracts. Les densités maxima atteignant $50 \%$ environ de la surface des parois, et l'épaisseur moyenne enlevée étant de $18,1 \mathrm{~mm}$, on peut estimer la masse maximum détachée à $22500 \mathrm{~g} / \mathrm{m}^{2}$ (à comparer avec les $181,5 \mathrm{~g} / \mathrm{m}^{2}$ fournis par la gélifraction sur les calcaires santoniens des collines de La Fare (à l'ouest d'Aix-en-Provence) au cours de l'hiver 1969-1970, (GABERT et al., 1981). En estimant les parois à $50 \%$ de la surface brûlée, et à $10 \%$ des parois la surface ignifractée, soit 250 ha, et en attribuant un taux moyen de $25 \%$ de la surface affectée par les enlèvements d'ignifracts, on peut envisager une ablation de l'ordre de 2800 tonnes de roche cohérente par ignifraction, avec une marge d'erreur de l'ordre de $200 \%$.

\section{CONCLUSIONS}

Cette première étude d'ignifracts, sommaire, se proposait principalement de mettre en évidence les caractéristiques très particulières de ces objets ainsi que l'importance considérable des tonnages libérés par l'ignifraction en quelques heures. L'existence d'ignifracts a été observée, à la suite d'incendies, sur les calcaires santoniens à l'ouest d'Aix-en-Provence par l'un d'entre nous (BALLAIS), sur les calcaires du Mont Carmel, en Israël (INBAR, communication orale), ainsi que sur les grès de Montserrat, en Espagne (MARQUES, communication orale).

Par contre, les gneiss et micaschistes du massif des Maures, en Provence cristalline, ne semblent pas avoir été affectés par l'ignifraction (observations de J.-L. BALLAIS). Différence dans la température du feu ou/et influence structurale ? De nouvelles recherches devront en décider, en particulier par le biais d'expérimentations concernant le rôle de la température sur la répartition, la densité, voire l'épaisseur des ignifracts.

Enfin, il est possible d'envisager maintenant une recherche des preuves d'incendie d'âge historique, par le biais des ignifracts, de progresser donc dans la connaissance du nombre des incendies récents. Mais il doit être également possible de s'interroger sur le rôle joué, à l'échelle de la période historique ou à l'échelle de la période holocène, par l'ignifraction dans la fragmentation mécanique des roches, en particulier dans le domaine méditerranéen : s'il est vrai que l'ignifraction est de 10 à 100 fois plus efficace que la gélifraction, ne peut-elle pas constituer l'origine principale de la genèse de nouveaux éclats ? C'est le problème que nous allons tenter de résoudre, sur la montagne Sainte-Victoire, par de nouvelles recherches en collaboration étroite avec des archéologues.

Remerciements : Nous voudrions remercier Christine KING, Moshe INBAR, Maria Angels MARQUES et Jan DE PLOEY pour les discussions fructueuses à propos des ignifracts.

\section{RÉFÉRENCES BIBLIOGRAPHIQUES}

BACCOUCHE N., (1990). - La forêt du massif de Sainte Victoire et les incendies : le cas de l' incendie de 1989, Mémoire D.E.A., Aix-Morseille II, 79 p.ronéot.

BOSC M.-C., (1990).- Les conséquences de l'incendie d'août 1989 sur les processus d'érosion dans le bassin-versant du Bayon, Mémoire de Maîtrise, Aix-Marseille II, 113 p. ronéot. 
GABERT P., MARRE A., RISER J., (1981).- Processus d'érosion avec intervention du gel sur des versants témoins en Basse-Provence intérieure (France), Méditerranée, Aix-en-Provence, 1, p. 13-20.

TRICART J., CAILlEUX A., (1955).- Initiation à l'étude des sables et des galets, 3 vol., SEDES, Paris. 